\title{
Letter of response to Professor Dalgleish regarding our paper Re: Long-term evaluation of anabolic and anti-resorptive agents in adults with familial osteoporosis due to pro205ala variant of the col1a1 gene
}

\author{
Stephen P. Tuck ${ }^{1,2}$ [D $\cdot$ Harish K. Datta ${ }^{3}$
}

Received: 2 February 2022 / Accepted: 4 February 2022 / Published online: 2 March 2022

(c) International Osteoporosis Foundation and National Osteoporosis Foundation 2022

Dear Sir/Madam,

We would like to thank Professor Dalgleish for his interest in our paper. In particular, we thank Professor Dalgleish for pointing out presentation errors on Fig. 1 in the supplementary tables and in the text that did not comply with the recommendations of the HUGO Gene Nomenclature Committee nor those of the Human Gene Variation Society sequence variant nomenclature. We would like to apologise for this inadvertent error due to our ignorance of their existence. We shall certainly make a point of using these should we publish in this area in the future.

Professor Dalgleish's main criticism centres on case 5 that had osteoporosis, but did not have the sequence variant and as such he argues that we have not demonstrated that the sequence variant is causative of a low bone density phenotype. However, case 5 has multiple secondary causes that explain his low bone density. He is of short stature which of itself is associated with low areal bone density as the DEXA scanner being two dimensional does not adjust for size differences. He also has a low body mass index of 16.7 ; he is a smoker and has growth hormone deficiency requiring growth hormone as a child and had periods of malnutrition. The genetic determinants of bone density are polygenic, and there are considerable lifestyle and environmental influences on bone density. Furthermore, phenotypic variability in conservative as well nonconservative amino acid substitution in collagen is well known both within and between affected families. This is seen in cases 1-4 who all have the sequence variant. We have provided

Stephen P. Tuck

stephen.tuck@nhs.net

1 Institute of Clinical and Translational Research, Medicine, Newcastle University, Framlington Place, Newcastle upon Tyne NE2 4HH, UK

2 Department of Rheumatology (S.P.T.), James Cook University Hospital, Middlesbrough TS4 3BW, UK

3 Department of Blood Sciences (Pathology), James Cook University Hospital, Middlesbrough TS4 3BW, UK numerous references in the paper to support this point. This includes papers describing this variant sequence (e.g. reference 16 Spotila et al. 1996) and others (e.g. Zhuang et al. reference 15 in 1996). The Zhuang paper from 1996 (reference 15) does indeed refer to a different sequence variant to the one discussed in our paper for which we apologise for our error. However, the point we are making in referencing that paper is to give an example of phenotypic variability in families with conserved substitutions, which supports our findings.

In answer to point 2 to regarding the asterisk on subject 3 , this error was carried over from previous versions of the paper. We had originally highlighted subjects with the sequence variant with an asterisk, but this seemed confusing as the subjects were all mixed in the table. It seemed better to order the table into groups rather than distinguish them by an asterisk. Hence, these were removed. We are sorry one asterisk got left. In answer to point 5, the method was in an earlier draft of the paper, but was edited out of the current version as it is a short paper, but inadvertently not removed from the text. In answer to point 5 and point 8 , the initial whole collagen sequence analysis on the index case was carried out in the NHS Genetic Diagnostic Laboratory (Sheffield, UK), and once the particular substitution was identified, all other samples were processed for that substitution in the same laboratory.

Once again, we thank Professor Dalgleish for his interest and have answered his points. We do not believe any of his criticisms alter the important messages of our paper. We have described an unusual sequence variant that predisposes to a low bone density phenotype. We also report long-term follow-up of these individuals with the effects of different treatments that have been tried. Our findings may well be of use should other people encounter families with the same sequence variant.

Yours sincerely.

Publisher's Note Springer Nature remains neutral with regard to jurisdictional claims in published maps and institutional affiliations. 